\section{New technique of railroading procedure for transnasal placement of devices following transoral endoscopy}

\section{Introduction}

Endoscopic guided placement of nasojejunal tube (NJT), nasogastric tube (NGT), nasobiliary drain (NBD) and nasopancreatic drain (NPD) is employed for enteral tube feeding, biliary drainage and pancreatic pseudocyst drainage, respectively. During railroading procedure, the final step is to reroute the endoscopically placed NJT/NGT/NBD through the anterior nares. In routine practice, for rerouting we use a tongue depressor and Magill forceps or employ transoral manipulation with fingers to retrieve the nasally placed Ryle's tube (RT) from the oropharynx. These procedures cause discomfort to the patient with chances of trauma to the posterior pharyngeal wall, as well as accidental bite injury to the technician/ endoscopist. To overcome these problems, we describe a novel technique in which there is decreased discomfort to the patient and no need to use any instrument or transoral finger manipulation by the technician/endoscopist.

\section{Technique}

The patient should be explained clearly about the whole procedure with special emphasis not to swallow the Ryle's tube when it enters into the oral cavity after insertion through anterior nares. Initially, measure the length of Ryle's tube from anterior nares to the tragus of corresponding ear. A large size RT $(16 \mathrm{~F}$ or $18 \mathrm{~F})$ is preferable as it is less flexible with less chance of loop formation in the oropharynx. Insert the Ryle's tube through the anterior nares up to a length equal to the distance between the anterior nares and the tragus of ipsilateral ear. Instruct the patient not to swallow the Ryle's tube, while applying gentle pressure over the cricoid cartilage with the assistance of a second person.

On further advancement, the Ryle's tube enters the oral cavity with or without loop formation (Figures $1 \& 2$ ). Thus, there is no need of any instrument (tongue depressor and Magill forceps) or transoral manipulation with fingers. Once the Ryle's tube reaches the oral cavity, trace its tip and attach it to an NJT, NBD or NGT which has already been placed using a transoral endoscope. After connecting both the tubes, gradually withdraw the RT so that it drags out the connected tube (NJT, NBD or NGT) through the nostril, and is finally fixed to the bridge of the nose.

\section{Discussion}

In endoscopic practice, when nasal endoscopy is not frequently available for placement of feeding or drainage tubes, we place an NGT, NJT or NBD by using a transoral endoscope and later

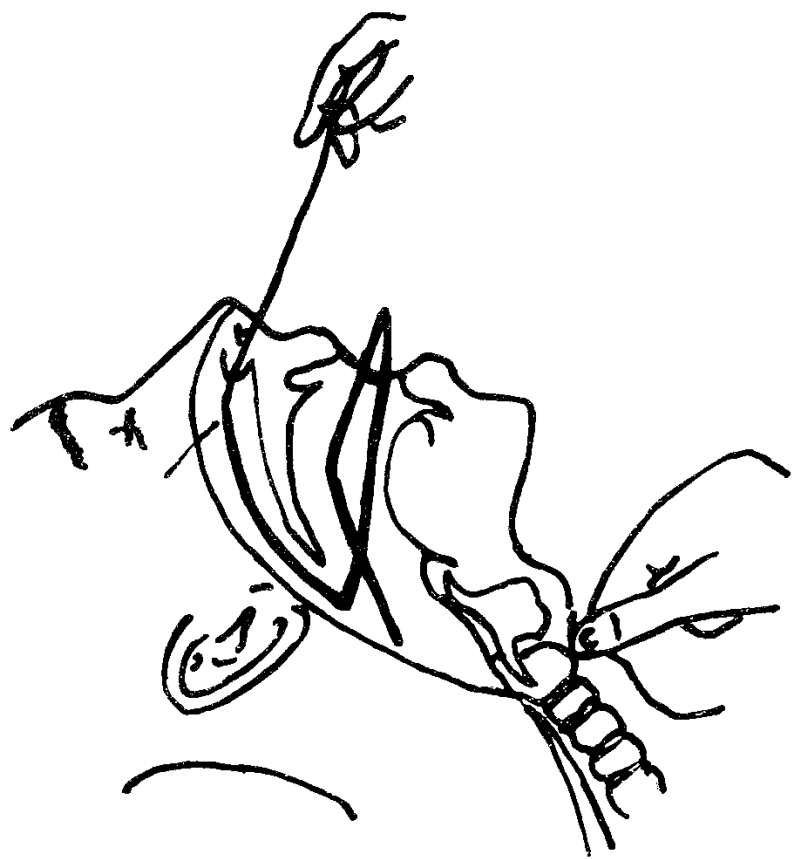

Figure 1: Nasally placed Ryle's tube coming out through the mouth by forming a loop. Cricoid pressure is applied while pushing the tube.

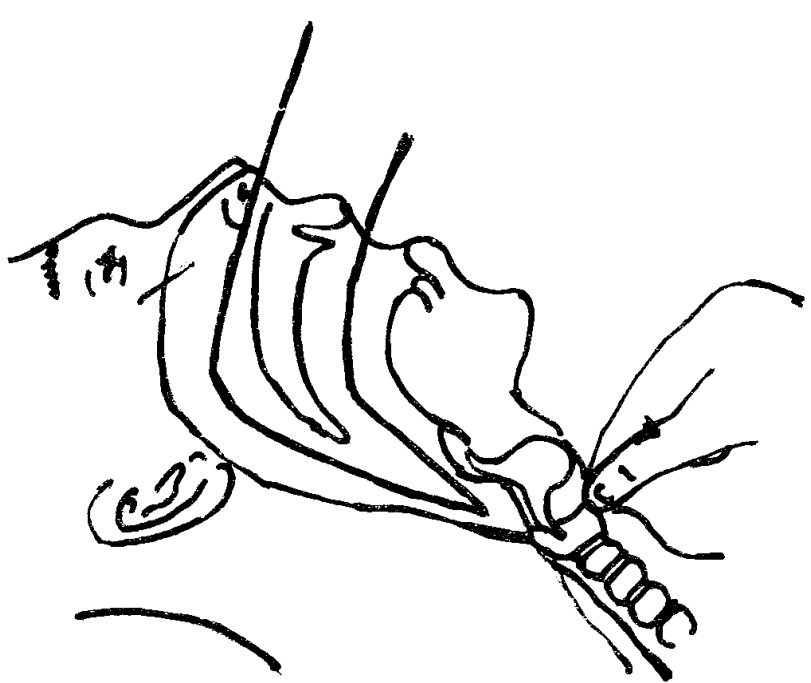

Figure 2: Nasally placed Ryle's tube coming out of the mouth without any loop formation 
rerouting the tube through the nose. While rerouting, we retrieve the nasally placed Ryle's tube from the posterior pharynx into oral cavity by using a tongue depressor and Magill forceps or by manipulating it with fingers. Both these procedures cause discomfort to patients and may traumatize the posterior pharyngeal wall.

In principle our technique avoids the passage of the nasally placed RT deep into the esophagus. This is achieved by instructing the patient not to swallow and by applying gentle external pressure on the cricoid cartilage. The anterior part of the cricoid cartilage forms an important landmark in front of the neck; it lies opposite the sixth cervical vertebra, and demarcates the junctions of pharynx with esophagus and larynx with trachea. ${ }^{1}$ Thus given its position above the pharyngoesophageal junction, external pressure on the cricoid cartilage compresses the upper esophageal sphincter against the vertebral column thus preventing the passage of RT deep into the esophagus. Further, by instructing the patient not to swallow and advancement of the nasally placed RT simultaneously causes redirection of the RT into the oral cavity with or without loop formation (Figures 1 \& 2). Once RT comes out through the oral cavity its tip is traced and attached to the NJT, NGT or NBD catheter already in place. Thereafter the nasally placed RT is pulled through the anterior nares which redirects the orally placed tube back through the nose.

This technique was prospectively applied on 30 patients, and was successful in 28 patients. One patient was extremely uncooperative, in whom the RT came over dorsum of tongue and the other patient needed transoral manipulations with fingers. None of the patients experienced aspiration or transtracheal passage of the tube. The advantages of this technique include, 1) less discomfort to the patient, 2) lower risk of posterior pharyngeal trauma, 3 ) avoids accidental bite injury to endoscopists, particularly with uncooperative patients, and 4) decreases cost and burden of sterilizing instruments.

YALAKA RAMI REDDY SURINDER SINGH RANA CHALAPATHI RAO DEEPAK KUMAR BHASIN

Correspondence: Dr. Surinder S Rana Department of Gastroenterology, Post Graduate Institute of Medical Education and Research, Sector 12, Chandigarh - 160012, India

Email:drsurinderrana@yahoo.co.in, sonalisurinder@yahoo.co.in

\section{References}

1. Benkhadra M, Lenfant F, Bry J, Astruc K, Trost O, Ricolfi F, et al. Cricoid cartilage and esophagus: CT scan study of the dynamic variability of their relative positions. Surg Radiol Anat. 2009;31:537-43.

\section{An unusual case of epidermoid- splenic cyst}

\section{Introduction}

Splenic cysts are uncommon entities with an incidence of $0.5-$ $2.0 \% .^{1}$ Acomprehensive differential diagnosis of cystic lesions of the spleen includes parasitic echinococcal cyst, congenital cyst, intersplenic pancreatic pseudocyst, pseudocystarising from splenic trauma,necrotising infection, pyogenic splenic abscess, metastatic diseases and cystic lymphangioma or hemangioma. ${ }^{2}$

\section{Case report}

A 35-year-old male presented with complaints of abdominal discomfort and pain left hypochondriumradiating to ipsilateralshoulder. There was no history of trauma and weight loss. Physical examination revealed a large palpable ,nonmobile, firm to hardswellingin the left hypochondrium. Routine investigations were normal. An ultrasonogram (USG) revealeda well defined cystic mass. On CT scan a $14 \times 12$ cm cyst was localized to the spleen which had displaced the splenic parenchyma andthe possibility of a hydatid cyst was entertained. Exploratory laparotomy with splenectomy was performed. Peroperatively a large cyst sized $14 \times 12 \mathrm{~cm}$ was seen. The splenectomyspecimen was received in our pathology department,weighing 550gm and measuring $15 \times 13 \times 4 \mathrm{~cm}$. Grossly, awell encapsulated cystic area along with normal splenic tissue was identified. On cut section, the cyst had a thick wall and contained brown colored fluid. The interior surface was trabeculated (Figure 1). Microscopic examination revealed a thick fibrous wall lined by squamous epithelium (Figure 2). Focal areas of calcification were also present. Immunohistochemistry was positive for cytokeratin (CK) (Figure 3) and negative for calretinin. The final diagnosis of a 